\title{
Ueber die Adsorption von
}

Natriumaurichlorid an Kohle und die Bestimmung des Goldes im Meerwasser

\section{Doctoral Thesis}

Author(s):

Koch, Hellmuth

Publication date:

1918

Permanent link:

https://doi.org/10.3929/ethz-a-000099611

Rights / license:

In Copyright - Non-Commercial Use Permitted 
Diss. ETH: $191 B$

\section{Ueber die Adsorption \\ von Natriumaurichlorid an Kohle \\ und die Bestimmung des Goldes im Meerwasser}

Von der

Eidgenössischen Technischen Hochschule

in Zürich

zur Erlangung der

Würde eines Doktors der technischen Wissenschaften

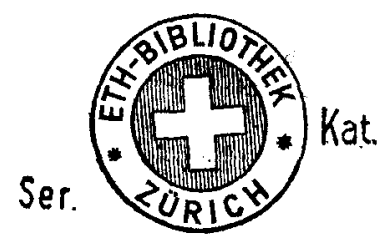

genehmigte

Promotionsarbeit

vorgelegt von

Hellmuth Koch, Diplom-Ingenieur

aus Zurich

Referent: Herr Prof. Dr. E. Ba u r

191

Korreferent: Herr Prof. Dr. F. P. Treadwell

DRESDEN und LEIPZIG 1918

Theodor Steinkopff, Verlagsbuchhandlung

- 
Leider können wir diese Angaben, die von F. W. Clarke in dem Werke: "Data of Geochemistry " (Washington, Gov. Print. Off. 1908) zitiert werden, nicht auf ihre Tragweite prüfen, da mir die Originalliteratur nicht zugänglich ist. $\mathrm{Da}$ aber Wagon e r's Werte mit den beiden vermutlich besten Angaben von Don und Münster nahe übereinstimmen, so darf man wohl alle drei als sich wechselseitig bestätigend ansehen.

Schließlich existiert noch eine Mitteilung des Goldprobierchemikers M. Pack aus San Franzisko, wonach Wasser des Pazifik einen Goldgehalt von weniger als $1 / 2$ grain $=32,4 \mathrm{mg}$ $\mathrm{Au} / \mathrm{cbm}$ enthalten soll.

Gegen diese Angaben wendet sich J. Loewy, der mit Wasser aus der Bucht von Biskaya arbeitend, nicht mehr als Bruchteile eines Milligramms Gold pro Kubikmeter finden konnte. $\mathrm{Da}$ weder die eine noch die andere Mitteilung erkennen läßt, mit welchen Wassermengen gearbeitet wurde und nach welchem Verfahren, so läßt sich über die erzielte Genauigkeit kein Urteil gewinnen und es erübrigt sich daher ein näheres Eingehen darauf.

Zusammenfassend läßt sich sagen, daßi den höheren von Sonstadt und Liversidge angegebenen Goldgehalten die niedrigeren zwischen 5 und $10 \mathrm{mg} \mathrm{Au} / \mathrm{cbm}$ sich bewegenden Befunde von $M$ ünster, Don und Wagoner gegenüberstehen, sowie die negativen von $\mathrm{de}$ Wilde in der Nordsee. Es ist durchaus möglich, daß verschiedene Meere verschiedene Goldmengen enthalten, namentlich $\mathrm{da}$, wo durch reichliche Zufuhr sedimentierenden Materials die Entgoldung ein dauernd wirkender Naturvorgang ist, kann ein negativer Befund durchaus als Bestätigung der Adsorptionserscheinung gebucht werden.

Meine eigenen Bestimmungen des Meerwassers von der Riviera und der Adria erscheinen niedrig, verglichen mit den Angaben aus den Weltmeeren. Man muß es aber als wünschenswert bezeichnen, dieselben mit grösseren Mengen, mindestens 100 Liter, zu wiederholen, ehe die Messungen als völlig befriedigend betrachtet werden können.

\section{Literatur.}

E. Sonstadt, On the Presence of Gold in Sea-water (Chem. News 26, 159, 1872).

C. A. Münster, On the possibility of Extracting the Precious Metals from Sea-Water (Journ. soc. chem. ind. 11, 351, Ref.; Norsk Tekniak Tidsskrift 10, Nr. 1 (1892), Orig.).

A. Liversidge, On the Amount of Gold and Silver in Sea - water (Journ. and Proc. Royal Soc. N.S.W. 29, 335, 1895.)
E. Sonstadt, The oxydation of Ferrous Sulphate by Sea-water and on the Detection of Gold in Seawater (Chem. News 74, 316, 1896).

A. Liversidge, Presence of Gold in Natural Saline, Deposits and Marine Plants (Journ. chem. soc. 71, 298, 1897).

J. R. Don, Die Entstehung von Goldgängen in Australien und Neuseeland (Zeitschr. f. prakt. Geol. 357, Ref.; Trans. Amer. Inst. Min. Eng. 27, 564 bis 668 (1897), Orig.)

M. Pack, L'extraction de l'or contenu dans l'eau de mer (Rev. scient. 11, 381, 1899).

L. Wa goner, Trans. Amer. Inst. Min. Eng. 31, 806 (1901).

P. de Wilde, Sur l'or contenu dans l'eau de la mer (Arch. sc. phys. nat. 19, 559, 1905).

K. Friedrich, Untersuchungen ïber den Goldgehalt von Gebirgsproben und Solen deutscher Salzlagerstätten (Metallurgie 3, 627, 1906).

J. Loewy, Gold im Meerwasser (Chem.-Ztg. 29, 213, 1905).

L. Wagoner, Trans. Amer. Inst. Min. Eng. 38, 704 (1907).

\section{Zusammenfassung.}

1. Es wird die Adsorptionsisotherme für Goldchlorid in dreiprozentiger Chlornatriumlösung an Tierkohle, Holzkohle, Koks, Tusche und KienruB bis zu den tiefsten erreichbaren Konzentrationen durchgemessen. Der äußerste gravimetrisch bestimmte Punkt liegt bei der Konzentration $18 \mathrm{mg} \mathrm{Au} / \mathrm{cbm}$.

2. Es wird der zeitliche Verlauf der Reduktion des adsorbierten Goldsalzes und die damit zusammenhängende Nachadsorption gemessen.

3. Es wird eine Methode ausgearbeitet, um durch Adsorption an Holzkohle kleinste Mengen gelöster Goldsalze, wie sie z. B. im Meerwasser vorhanden sind, genau zu bestimmen.

4. Die vorhandenen Angaben über den Goldgehalt des Meerwassers werden zusammengestellt und kritisch gesichtet.

Vorliegende Arbeit wurde auf Anregung und unter Leitung von Prof. Dr. E. B a u r im physikalisch - chemischen Institut der eidgenössischen technischen Hochschule ausgeführt. Es sei mir an dieser Stelle gestattet, meinem hochverehrten Lehrer für seine wertvolle Unterstützung und sein stets reges Interesse meinen aufrichtigsten Dank auszusprechen.

Auch Herrn Prof. Dr. F. P. Treadwell, der mir in bereitwilligster Weise die Spezialeinrichtungen des Goldprobierlaboratoriums zur Verfügung stellte, möchte ich meinen besten Dank bezeugen.

Zürich, im Oktober 1917.

$$
\begin{aligned}
& \text { Laboratorium für physikalische Chemie } \\
& \text { der Eidg. Techn. Hochschule. }
\end{aligned}
$$

